\title{
Surface electronic structure of the wide band gap topological insulator $\mathrm{PbBi}_{4} \mathrm{Te}_{4} \mathrm{Se}_{3}$
}

\author{
I. A. Shvets,,${ }^{1,2}$ I. I. Klimovskikh, ${ }^{2}$ Z. S. Aliev,${ }^{3,4}$ M. B. Babanly,,${ }^{5,6}$ F. J. Zúñiga, ${ }^{7}$ J. Sánchez-Barriga,${ }^{8}$ M. Krivenkov, ${ }^{8}$ \\ A. M. Shikin, ${ }^{2}$ and E. V. Chulkov ${ }^{9,10,2,1}$ \\ ${ }^{1}$ Tomsk State University, 634050 Tomsk, Russia \\ ${ }^{2}$ St. Petersburg State University, 198504 Saint Petersburg, Russia \\ ${ }^{3}$ Azerbaijan State Oil and Industry University, AZ1010 Baku, Azerbaijan \\ ${ }^{4}$ Institute of Physics, ANAS, AZ1143 Baku, Azerbaijan \\ ${ }^{5}$ Institute of Catalysis and Inorganic Chemistry, ANAS, AZ1143 Baku, Azerbaijan \\ ${ }^{6}$ Baku State University, AZ1148 Baku, Azerbaijan \\ ${ }^{7}$ Departamento de Física de la Materia Condensada Facultad de Ciencia y Tecnología, Universidad del País Vasco, E-48080 Bilbao, Spain \\ ${ }^{8}$ Helmholtz-Zentrum Berlin für Materialien und Energie, Elektronenspeicherring BESSY II, \\ Albert-Einstein-Strasse 15, 12489 Berlin, Germany \\ ${ }^{9}$ Departamento de Física de Materiales UPV/EHU, Centro de Física de Materiales CFM - MPC and Centro Mixto CSIC-UPV/EHU, \\ 20080 San Sebastián/Donostia, Basque Country, Spain \\ ${ }^{10}$ Donostia International Physics Center (DIPC), 20018 San Sebastián/Donostia, Basque Country, Spain
}

(Received 30 January 2019; published 18 November 2019)

\begin{abstract}
By means of angle-resolved photoemission spectroscopy (ARPES) measurements and density functional theory (DFT) calculations, the electronic band structure of the topological insulator $\mathrm{PbBi}_{4} \mathrm{Te}_{4} \mathrm{Se}_{3}$ for both five-layer and seven-layer surface terminations is investigated. The measured and calculated band structure features are in good agreement and indicate two well-resolved topological surface states with distinct spatial localizations within bulk band gap of about $0.3 \mathrm{eV}$.
\end{abstract}

DOI: 10.1103/PhysRevB.100.195127

\section{INTRODUCTION}

Three-dimensional topological insulators (TIs), characterized by the nontrivial bulk band topology due to a strong spin-orbit interaction, exhibit an odd number of gapless spinhelical topological surface states (TSSs) in the bulk energy gap $[1,2]$. These states are protected by the time reversal symmetry and, as a result, remain gapless under a nonmagnetic perturbation. Due to unique properties, TSSs are of great interest for the observation of many novel quantum phenomena, in particular, quantum spin Hall effect and quantum anomalous Hall effect [3].

So far, a wide variety of compounds hosting spin-polarized TSSs have been thoroughly studied [4-22]. After the discovery of narrow-gap tetradymite-type semiconductors with quintuple-layer (QL) crystal structure [4-6], further efforts at controlling the electronic structure of TIs and TSSs led to fabrication and study of septuple-layer (SL) compounds [7-9]. Another way of controlling the electronic structure of TIs involves the isovalent substitution of atoms within sublattice, resulting in formation of alloys with fractional stoichiometry [10-15].

Recently, a series of the heterostructures $\left[\mathrm{PbBi}_{4} \mathrm{Te}_{7}\right.$, $\left.\mathrm{PbBi}_{6} \mathrm{Te}_{10}, \mathrm{Sn}(\mathrm{Bi}, \mathrm{Sb})_{4} \mathrm{Te}_{7}\right]$ formed by alternating $\mathrm{QL}$ $\left(\mathrm{X}_{2} \mathrm{Te}_{3}\right)$ and $\mathrm{SL}\left(\mathrm{YX}_{2} \mathrm{Te}_{4}\right)(\mathrm{X}=\mathrm{Bi}, \mathrm{Sb} ; \mathrm{Y}=\mathrm{Pb}, \mathrm{Sn})$ blocks of TIs have been theoretically proposed $[9,10,16-18]$ and experimentally verified $[9,19,20]$. In such a case, terraces with nonequivalent 5L- and 7L-surface terminations are possible after crystal cleavage. In the experiment by using ARPES methods, a few Dirac cones can be simultaneously observed.
For instance, in $\mathrm{PbBi}_{4} \mathrm{Te}_{7}$ [9,19] (with 5L and 7L terminations) and $\mathrm{PbBi}_{6} \mathrm{Te}_{10}$ [20] (with 7-5L, 5-7L, and 5-5L terminations) two and three TSSs, respectively, have been revealed. According to theoretical studies, all these compounds show narrow energy gap up to $150 \mathrm{meV}$ as well as entangled spatial charge density distribution of the TSS, localized within a thick surface region.

On the other hand, the aforementioned heterostructures are tellurium-based compounds. Very recently studied sulfurcontaining $\mathrm{PbBi}_{4} \mathrm{Te}_{4} \mathrm{~S}_{3}$ has a significantly larger band gap $(\sim 0.2 \mathrm{eV}$ in experiment and $\sim 0.3 \mathrm{eV}$ in theory) $[21,22]$ and exhibits the topological nature of the surface states. Another candidate for isovalent substitution is the Se atom. As demonstrated in previous works, the partial substitution of the heavy chalcogen atom Te by Se in TIs leads to advantageous modification of the electronic structure. Namely, as for the QL constituent, the experimentally measured bulk band gap for $\mathrm{Bi}_{2} \mathrm{Te}_{3}$ is $165-170 \mathrm{meV}$ [4,5], while for $\mathrm{Bi}_{2} \mathrm{Te}_{2} \mathrm{Se}$ it is $\sim 0.3 \mathrm{eV}$ [10]. As was shown theoretically in Ref. [14], in the $\mathrm{SL} \mathrm{PbBi}_{4} \mathrm{Te}_{4-x} \mathrm{Se}_{x}$ compound the substitution of Te by $\mathrm{Se}$, in addition to a significant increase of the bulk band gap (from $90 \mathrm{meV}$ for $x=0$ up to $220 \mathrm{meV}$ for $x=2$ ), leads to redistribution of the charge density of TSS, shrinking its depth profile. Thus, in the heterostructures, containing alternating QL and SL with Se content, the band gap is expected to be larger and TSS to be localized closer to the surface with shorter penetration length. As a consequence, such materials might be suitable for realization of the thin-film systems based on TIs, avoiding hybridization between the Dirac-like surface states arising at the opposite surfaces. 
TABLE I. Experimental structure parameters in relative coordinates for $\mathrm{PbBi}_{4} \mathrm{Te}_{4} \mathrm{Se}_{3}$ with $a=4.3224 \AA$ and $c=23.3725 \AA$.

\begin{tabular}{lccc}
\hline \hline Atom & $x$ & $y$ & $z$ \\
\hline $\mathrm{Pb}$ & 0 & 0 & 0.00000 \\
$\mathrm{Bi} 1$ & $1 / 3$ & $2 / 3$ & 0.15847 \\
$\mathrm{Bi} 2$ & $1 / 3$ & $2 / 3$ & 0.42031 \\
$\mathrm{Te} 1$ & 0 & 0 & 0.23213 \\
$\mathrm{Te} 2$ & $2 / 3$ & $1 / 3$ & 0.34613 \\
$\mathrm{Se} 1$ & 0 & 0 & 0.50000 \\
$\mathrm{Se} 2$ & $2 / 3$ & $1 / 3$ & 0.07406 \\
\hline \hline
\end{tabular}

In the present work, we examine the electronic band structure of $\mathrm{PbBi}_{4} \mathrm{Te}_{4} \mathrm{Se}_{3}$ in terms of ARPES measurements and DFT calculations. We discuss main peculiarities of the electronic structure and provide analysis of the charge density distribution of TSSs.

\section{EXPERIMENTAL AND COMPUTATIONAL DETAILS}

The $\mathrm{PbBi}_{4} \mathrm{Te}_{4} \mathrm{Se}_{3}$ compound [Fig. 1(a)] has a trigonal crystal structure (group $P \overline{3} m 1$ ) formed by alternating $\mathrm{Bi}_{2} \mathrm{Te}_{2} \mathrm{Se} \mathrm{QL}$ and $\mathrm{PbBi}_{2} \mathrm{Te}_{2} \mathrm{Se}_{2} \mathrm{SL}$ blocks. The two adjacent blocks are weakly bonded to each other by the van der Waals (vdW) forces, whereas the bonding between atoms within each block is determined by ionic-covalent interaction. Therefore, the crystal tends to cleave at the vdW gap leading to appearance of different possible surface terminations.

The $n$-doped sample with stoichiometry of $\mathrm{PbBi}_{4} \mathrm{Te}_{4} \mathrm{Se}_{3}$ was grown from the high-purity elements $(\mathrm{Pb}, \mathrm{Bi}, \mathrm{Te}, \mathrm{Se})$ by a modified vertical Bridgman method. A powdered sample for $\mathrm{X}$-ray diffraction (PXRD) measurements was prepared using selected cleaved platelets from the ingot. The collected PXRD pattern was used to carry out a Rietveld refinement using the structural data of $\mathrm{PbBi}_{4} \mathrm{Te}_{7}$ [23] as a model. Details of the phase diagram and structural details are under preparation to be published elsewhere. The refined lattice parameters are given in Table I. As can be seen in Fig. 1(a), comparing with the structure of $\mathrm{PbBi}_{4} \mathrm{Te}_{7}$ the $\mathrm{Se}$ atoms replace Te in the internal layers of the QL and SL blocks. For the photoemission measurements in ultrahigh vacuum with the base pressure of $1-2 \times 10^{-10}$ mbar, the single-crystalline sample was cleaved in situ from the same ingot. The ARPES experiment was carried out at the RGBL-2 end station at room temperature using linearly-polarized undulator radiation at the synchrotron source BESSY-II in Berlin.

The electronic structure calculations were performed within the density functional theory implemented in VASP code $[24,25]$. We employed the all-electron projector augmented wave [26,27] basis sets with the generalized gradient approximation [28] to the exchange correlation potential. Relativistic effects, including spin-orbit coupling, were taken into account. Experimental lattice parameters and atomic positions used in the study are presented in Table I. We used the $7 \times 7 \times$ 7 and $9 \times 9 \times 1 k$-point grids for bulk and slab self-consistent calculation, respectively. To simulate the surfaces, we used symmetric slabs with thickness of 41 (53) and 43 (55) atomic layers for 5L and 7L terminations, respectively. The vacuum space between slabs was set to $15 \AA$.

\section{RESULTS AND DISCUSSION}

In order to study the surface electronic structure of $\mathrm{PbBi}_{4} \mathrm{Te}_{4} \mathrm{Se}_{3}$, we have performed ARPES measurement using different photon energies (16, 25, and $60 \mathrm{eV})$. On ARPES spectra along the $\bar{\Gamma}-\bar{M}$ direction [Fig. 1(c)] one can see two distinct linearlike upward dispersing states resembling a Dirac cone (marked as DC1 and DC2), having minimum at the $\bar{\Gamma}$ point. By extrapolation of DC1 and DC2 to the Brillouinzone center the apexes can be defined at binding energies of $\sim 0.5$ and $\sim 0.7 \mathrm{eV}$, respectively. Note that the binding energies of DCs are slightly varied with photon energy due to photoemission-induced gating effects [29]. In order to see the band dispersion more clearly, the second derivatives of spectra of Fig. 1(c) are presented in Fig. 1(d). As seen, both states disperse parallel to each other with the energy shift of $\sim 0.15 \mathrm{eV}$. According to our DFT calculations [see Fig. 1(b)] and previous theoretical and experimental results for other systems $\left(\mathrm{PbBi}_{4} \mathrm{Te}_{7}\right.$ [9,19], $\mathrm{Sn}(\mathrm{Bi}, \mathrm{Sb})_{4} \mathrm{Te}_{7}$ [17], $\mathrm{PbBi}_{4} \mathrm{Te}_{4} \mathrm{~S}_{3}$ [22]), the "inner" state DC1, lying closer to the Fermi level, is associated with TSS originated from the 7L-terminated surface, while another "outer" state DC2 can be regarded as TSS induced by the 5L-terminated surface. As for the photoemission intensity, DC1 is less pronounced at low photon energies. At the same time DC2 has almost uniform intensity at nonzero momenta $k_{\|}$at all photon energies and only at $h v=60 \mathrm{eV}$ in $\bar{\Gamma}$ point the intensity is significantly enhanced. Comparing to ARPES measurements of the parent $\mathrm{PbBi}_{4} \mathrm{Te}_{7}$ [19] without Se content, where 7L and 5L blocks are represented by $\mathrm{PbBi}_{2} \mathrm{Te}_{4}$ and $\mathrm{Bi}_{2} \mathrm{Te}_{3}$, respectively, the state analogous to DC2 had been seen only at very low energy $h v=8.4 \mathrm{eV}$, allowing us to study deeper layers, regarding it as a buried TSS on the 5L-terminated surface.

Besides the discussed states DC1 and DC2, at lower photon energy (especially at $h v=25 \mathrm{eV}$ ) the diffuse M-shaped valence band state with an energy maximum of $E_{B} \approx 0.5 \mathrm{eV}$ and additional states in the energy region $0-0.25 \mathrm{eV}$ at the Brillouin-zone center become more intensive. The latter exhibit a paraboliclike dispersion with momentum offset bearing a resemblance to states with Rashba splitting. As was shown in Ref. [30], the underlying mechanism that causes the emergence of Rashba states is an expansion of vdW gaps induced by imperfections/defects trapped between neighboring blocks during the growth process. These states most likely overlap with the bulk conduction band states. Similar behavior of surface and bulk conduction bands had been observed in $\mathrm{PbBi}_{2} \mathrm{Te}_{4}$ [8] and $\mathrm{PbBi}_{4} \mathrm{Te}_{4} \mathrm{~S}_{3}$ [22]. Using this assumption one can speculate that the experimental bulk band gap of $\mathrm{PbBi}_{4} \mathrm{Te}_{4} \mathrm{Se}_{3}$ is about $0.3 \mathrm{eV}$. The calculated band gap is of $270 \mathrm{meV}$ [Fig. 1(b)], which is notably larger than those computed for $\mathrm{SbBi}_{4} \mathrm{Te}_{7}(75-100 \mathrm{meV})[17,18]$ and $\mathrm{PbBi}_{4} \mathrm{Te}_{7}$ (100 meV) [19]. The edges of the band gap are formed along the $\bar{\Gamma}-\bar{M}$ direction and, according to the well-known problem of the underestimation of the band gap width obtained in terms of DFT, the many-body correction calculations using, for example, the $G W$ approximation can overcome this issue and 

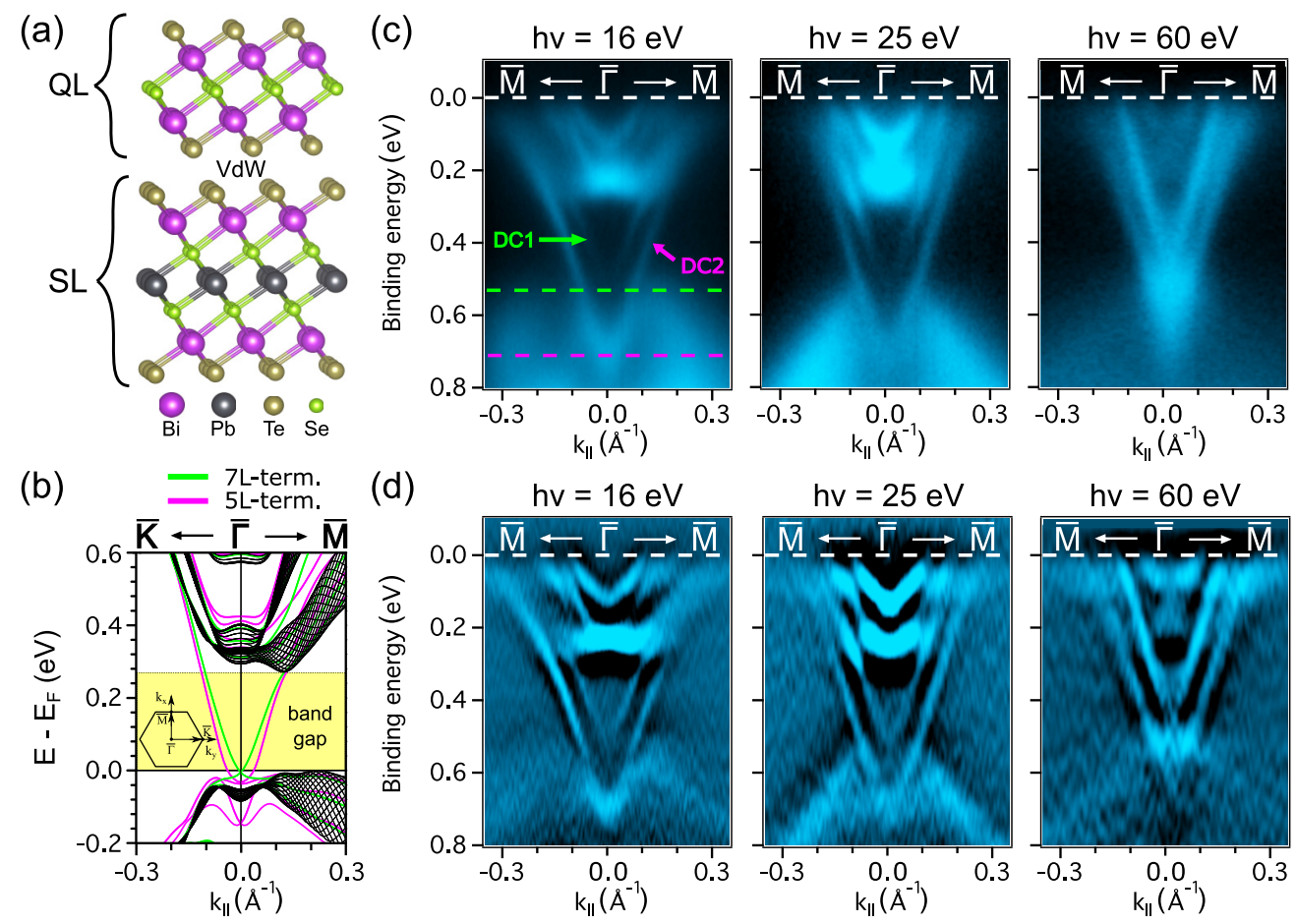

FIG. 1. (a) Crystal structure of $\mathrm{PbBi}_{4} \mathrm{Te}_{4} \mathrm{Se}_{3}$. (b) Calculated band structure of the $\mathrm{PbBi}_{4} \mathrm{Te}_{4} \mathrm{Se}_{3}(0001)$ thin films along $\overline{\mathrm{K}}-\bar{\Gamma}-\overline{\mathrm{M}}$ direction with seven and five-layer block terminations aligned to the bulk-projected bands (black curves), here the Fermi level is set to the energy of valence band maximum. The inset shows the high-symmetry points of the surface Brillouin zone. (c) Band dispersions of the $\mathrm{PbBi}_{4} \mathrm{Te}_{4} \mathrm{Se}_{3}$ sample along $\bar{\Gamma}-\bar{M}$ direction observed with ARPES measurement at various photon energies and (d) the second derivative images of these spectra. Horizontal dashed lines in (c) indicate the energy level for DC1 and DC2 at $\bar{\Gamma}$.

bring the theoretical band gap value closer to the experimental one [31].

In terms of the DFT calculation study, first we checked the size effect of the TI film on the energy dispersion by simulating the thin film with thickness up to 53 (55) atomic layers for the $5 \mathrm{~L}(7 \mathrm{~L})$-slab calculations. No notable quantitative difference between 41 (43) and 53 (55)-layered systems was observed, demonstrating the absence of hybridization between the TSSs from opposite surfaces. The latter is explicitly shown in the analysis of the spatial charge density distribution (Fig. 2). Hence, hereafter, we introduce and discuss the results obtained for the thinner films.

The calculated spectra of the $\mathrm{PbBi}_{4} \mathrm{Te}_{4} \mathrm{Se}_{3}$ slabs in Fig. 1(b) present dispersions for both 5L- (pink lines) and 7L-terminated (green lines) surfaces along the $\overline{\mathrm{K}}-\bar{\Gamma}-\overline{\mathrm{M}}$ direction aligned with the bulk-projected bands. In the fundamental band gap there are two TSSs with the Dirac cone-type behavior: the 7L-termination induced TSS with Dirac point (DP) at the valence band maximum energy and TSS originated from the 5L-terminated surface with DP $\sim 40 \mathrm{meV}$ below, being in close proximity to the bulk band. As a result, the latter remarkably deviates from linearity at $\bar{\Gamma}$ and the slope at larger $k_{\|}$is a little steeper. Along the $\bar{\Gamma}$ - $\bar{M}$ direction both TSSs merge into the conduction bulk band at $k_{\|} \approx 0.13 \AA^{-1}$. Another feature of the 5L-terminated surface spectrum is the occupied Rashba-type surface state which lies at $0.15 \mathrm{eV}$ below the Fermi energy in the local gap between the first and second bulk valence bands. Similar surface state had also been observed in other theoretical calculations for $\mathrm{Sn}(\mathrm{Sb}, \mathrm{Bi})_{4} \mathrm{Te}_{7}$,
$\mathrm{PbBi}_{4} \mathrm{Te}_{7}, \mathrm{PbBi}_{6} \mathrm{Te}_{10}$ and regarded as a survived TSS from the QL block. Comparing the theoretical and experimental spectra, the energy shift between DPs as well as the slope of the 7L TSS in the fundamental gap and 5L surface state in the local band gap are in a good agreement with those of DC1 and DC2.

In order to explain the experimental dispersion relations [Figs. 1(c) and 1(d)] and their discrepancy with the photoemission data for $\mathrm{PbBi}_{4} \mathrm{Te}_{7}$ [9,19], we conduct the detailed theoretical analysis of localization of the surface states. As seen in Fig. 2, near $\bar{\Gamma}$ the first bulk valence band $\left(E_{B}=\right.$ $-60 \mathrm{meV}$ ) is localized substantially in the SL subsurface block, while the second valence band $\left(E_{B}=-300 \mathrm{meV}\right)$ is formed in the QL blocks. It is important to note that the TSS charge density distributions of the $7 \mathrm{~L}$ and $5 \mathrm{~L}$ terminations qualitatively differ. In the case of 7L surface termination the TSS is almost entirely localized in the surface SL and at any $k_{\|}$the spatial distribution is similar to the profile calculated at the $\bar{\Gamma}$ point (point "a" in Fig. 2).

In the case of the $5 \mathrm{~L}$ termination, in the vicinity of $\bar{\Gamma}$ (point "b") TSS is mostly localized within the subsurface SL block. However, moving upward along both $\bar{\Gamma}-\bar{K}$ and $\bar{\Gamma}-\bar{M}$ directions, the weight of the TSS in the subsurface block decreases and, for example, at point "c" it is evenly distributed within two topmost blocks. For this reason we do not observe the actual DP of the 5L TSS as well as the region "b"-"c" by ARPES. In contrast to $\bar{\Gamma}-\overline{\mathrm{K}}$, where at larger $k_{\|}$(see point "e") TSS completely relocates to the surface QL, in the $\bar{\Gamma}-\bar{M}$ direction a measurable weight of TSS 

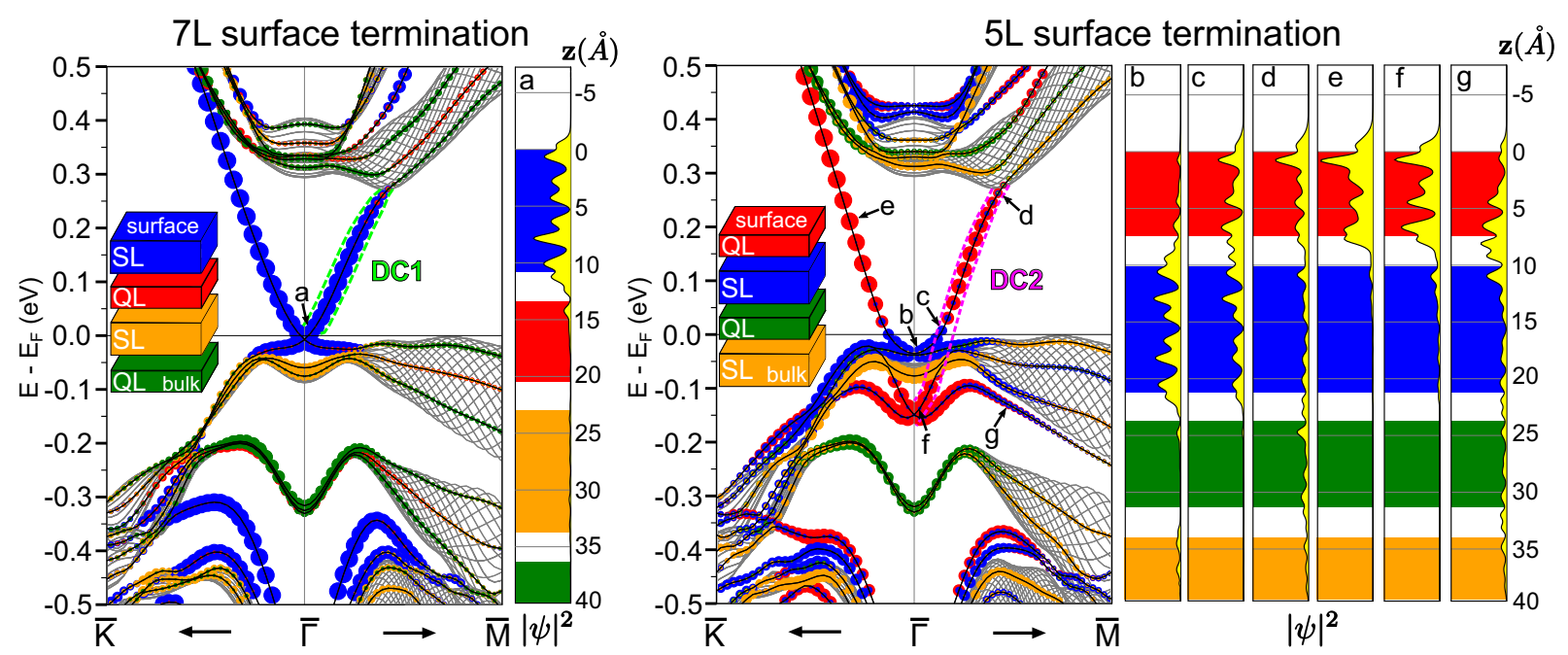

FIG. 2. Calculated band structure of $\mathrm{PbBi}_{4} \mathrm{Te}_{4} \mathrm{Se}_{3}$ surfaces along the $\overline{\mathrm{K}}-\bar{\Gamma}-\overline{\mathrm{M}}$ direction with $7 \mathrm{~L}$ and $5 \mathrm{~L}$ terminations; the color of the circles denotes surface/subsurface building blocks in the slabs, as shown in the inset, and size represents weights of the states in these layers; shaded area identifies the bulk-projected bands. Right subpanels (a)-(g) show charge density of the TSS integrated over $x, y$ at the respective $k$ points marked on spectra.

remains in the subsurface block, until merging into bulk states near "d". By comparison, in earlier studied $\mathrm{PbBi}_{4} \mathrm{Te}_{7}$ [19], where the corresponding TSS has more complicated spatial distribution along the $\bar{\Gamma}-\bar{M}$ direction, the band structure of the upper cone is kinklike and not distinctly defined, whereas in our investigation DC2 is linear and well observed. Finally, behavior of the Rashba-type occupied surface state, lying in the local gap, is quite opposite: in the center of the Brillouin zone ("f") it is predominantly located in the surface QL and, moving away from $\bar{\Gamma}$, the weight of the TSS in the deeper atomic layers increases. Also the slope of its upper branch is similar to that of the region "c"-"d". Thus, as we believe, we see this state as DC2 in the vicinity of $\bar{\Gamma}$ at $\sim 0.7 \mathrm{eV}$ on ARPES spectra [Figs. 1(c) and 1(d)].

\section{CONCLUSION}

In conclusion, by means of ARPES measurements and $a b$ initio calculations the surface electronic structure of the topological insulator $\mathrm{PbBi}_{4} \mathrm{Te}_{4} \mathrm{Se}_{3}$ has been studied. On the photoemission spectra due to the presence of terraces with two distinct terminations two Dirac conelike surface states are observed in the bulk gap. As shown by DFT calculations the inner state with energy minimum at binding energy of $\sim 0.5 \mathrm{eV}$ is the TSS originated from the seven-layer block termination and localized entirely in this block. While the outer state with apex at $\sim 0.7 \mathrm{eV}$ stems from the five-layer block termination and shows the nonuniform charge density distribution depending on momentum $k$. As calculations reveal, at larger values it is mainly contributed by TSS, emerging in the fundamental gap, however, its DP is buried and not observed in the ARPES images. Instead, photoemission intensity, measured at the minimum of the outer state, belongs to the Rashba-like surface state, arising in the local band gap. Among the known TIs formed by the alternating QL and SL blocks to this moment, the $\mathrm{PbBi}_{4} \mathrm{Te}_{4} \mathrm{Se}_{3}$ compound has the largest band gap and the shortest localization length of TSSs that can be useful for thin-film applications.

\section{ACKNOWLEDGMENTS}

We thank S. V. Eremeev for valuable discussions. This work is supported by the Russian Science Foundation (Grants No. 18-12-00169 in part of the density functional calculations and No. 18-12-00062 in part of the photoemission measurements) and Saint Petersburg State University (Grant ID 40990069). The support from the Academic D.I. Mendeleev Fund Program of Tomsk State University (Project No. 8.1.01.2018), the Russian Foundation for Basic Researches (Grant No. 18-52-06009), the Science Development Foundation under the President of the Republic of Azerbaijan (Grant No. EIF/MQM/Elm-Tehsil-1-2016-1(26)-71/01/4-M33), the Basque Country Government, Departamento de Educación, Universidades e Investigación (Grants No. IT-756-13 and No. IT1301-19) and the Spanish Ministerio de Ciencia e Innovación (Grant No. FIS2016-75862-P) are acknowledged. J.S.-B. gratefully acknowledges financial support from the Impuls-und Vernetzungsfonds der Helmholtz-Gemeinschaft under Grant No. HRSF-0067 (Helmholtz-Russia Joint Research Group). Calculations were partly performed using computational resources provided by Resource Center "Computer Center of SPbU" (http://cc.spbu.ru) and the SKIFCyberia supercomputer at the National Research Tomsk State University.
[1] A. Bansil, H. Lin, and T. Das, Rev. Mod. Phys. 88, 021004 (2016).

[2] Y. Ando, J. Phys. Soc. Jpn. 82, 102001 (2013).
[3] J. Sinova, S. O. Valenzuela, J. Wunderlich, C. H. Back, and T. Jungwirth, Rev. Mod. Phys. 87, 1213 (2015). 
[4] Y. L. Chen, J. G. Analytis, J.-H. Chu, Z. K. Liu, S.-K. Mo, X. L. Qi, H. J. Zhang, D. H. Lu, X. Dai, Z. Fang, S. C. Zhang, I. R. Fisher, Z. Hussain, and Z.-X. Shen, Science 325, 178 (2009).

[5] Y. Li, G. Wang, X. Zhu, M. Liu, C. Ye, X. Chen, Y. Wang, K. He, L. Wang, X. Ma, H. Zhang, X. Dai, Z. Fang, X. Xie, Y. Liu, X. Qi, J. Jia, S. Zhang, and Q. Xue, Adv. Mater. 22, 4002 (2010).

[6] Y. Xia, D. Qian, D. Hsieh, L. Wray, A. Pal, H. Lin, A. Bansil, D. Grauer, Y. S. Hor, R. J. Cava, and M. Z. Hasan, Nat. Phys. 5, 398 (2009).

[7] H. Jin, J.-H. Song, A. J. Freeman, and M. G. Kanatzidis, Phys. Rev. B 83, 041202(R) (2011).

[8] K. Kuroda, H. Miyahara, M. Ye, S. V. Eremeev, Yu. M. Koroteev, E. E. Krasovskii, E. V. Chulkov, S. Hiramoto, C. Moriyoshi, Y. Kuroiwa, K. Miyamoto, T. Okuda, M. Arita, K. Shimada, H. Namatame, M. Taniguchi, Y. Ueda, and A. Kimura, Phys. Rev. Lett. 108, 206803 (2012).

[9] S. V. Eremeev, G. Landolt, T. V. Menshchikova, B. Slomski, Yu. M. Koroteev, Z. S. Aliev, M. B. Babanly, J. Henk, A. Ernst, L. Patthey, A. Eich, A. Ako Khajetoorians, J. Hagemeister, O. Pietzsch, J. Wiebe, R. Wiesendanger, P. M. Echenique, S. S. Tsirkin, I. R. Amiraslanov, J. H. Dil, and E. V. Chulkov, Nat. Commun. 3, 635 (2012).

[10] M. Neupane, S.-Y. Xu, L. A. Wray, A. Petersen, R. Shankar, N. Alidoust, Ch. Liu, A. Fedorov, H. Ji, J. M. Allred, Y. S. Hor, T.-R. Chang, H.-T. Jeng, H. Lin, A. Bansil, R. J. Cava, and M. Z. Hasan, Phys. Rev. B 85, 235406 (2012).

[11] T. Arakane, T. Sato, S. Souma, K. Kosaka, K. Nakayama, M. Komatsu, T. Takahashi, Z. Ren, K. Segawa, and Y. Ando, Nat. Commun. 3, 636 (2012).

[12] S. Muff, F. von Rohr, G. Landolt, B. Slomski, A. Schilling, R. J. Cava, J. Osterwalder, and J. H. Dil, Phys. Rev. B 88, 035407 (2013).

[13] W. Ko, I. Jeon, H. W. Kim, H. Kwon, S.-J. Kahng, J. Park, J. S. Kim, S. W. Hwang, and H. Suh, Sci. Rep. 3, 2656 (2013).

[14] I. A. Shvets, I. I. Klimovskikh, Z. S. Aliev, M. B. Babanly, J. Sánchez-Barriga, M. Krivenkov, A. M. Shikin, and E. V. Chulkov, Phys. Rev. B 96, 235124 (2017).

[15] M. V. Filianina, I. I. Klimovskikh, I. A. Shvets, A. G. Rybkina, A. E. Petukhova, E. V. Chulkov, V. A. Golyashov, K. A. Kokh, O. E. Tereshchenko, C. Polley, T. Balasubramanian, M. Leandersson, and A. M. Shikin, Mater. Chem. Phys. 207, 253 (2018).
[16] S. V. Eremeev, Yu. M. Koroteev, and E. V. Chulkov, JETP Lett. 92, 161 (2010).

[17] M. G. Vergniory, T. V. Menshchikova, I. V. Silkin, Yu. M. Koroteev, S. V. Eremeev, and E. V. Chulkov, Phys. Rev. B 92, 045134 (2015).

[18] M. G. Vergniory, T. V. Menshchikova, S. V. Eremeev, and E. V. Chulkov, Appl. Surf. Sci. 267, 146 (2013).

[19] T. Okuda, T. Maegawa, M. Ye, K. Shirai, T. Warashina, K. Miyamoto, K. Kuroda, M. Arita, Z. S. Aliev, I. R. Amiraslanov, M. B. Babanly, E. V. Chulkov, S. V. Eremeev, A. Kimura, H. Namatame, and M. Taniguchi, Phys. Rev. Lett. 111, 206803 (2013).

[20] M. Papagno, S. V. Eremeev, J. Fujii, Z. S. Aliev, M. B. Babanly, S. Kr. Mahatha, I. Vobornik, N. T. Mamedov, D. Pacilé, and E. V. Chulkov, ACS Nano 10, 3518 (2016).

[21] I. V. Silkin, T. V. Menshchikova, M. M. Otrokov, S. V. Eremeev, Yu. M. Koroteev, M. G. Vergniory, V. M. Kuznetsov, and E. V. Chulkov, JETP Lett. 96, 322 (2012).

[22] K. Sumida, T. Natsumeda, K. Miyamoto, I. V. Silkin, K. Kuroda, K. Shirai, S. Zhu, K. Taguchi, M. Arita, J. Fujii, A. Varykhalov, O. Rader, V. A. Golyashov, K. A. Kokh, O. E. Tereshchenko, E. V. Chulkov, T. Okuda, and A. Kimura, Phys. Rev. Mater. 2, 104201 (2018).

[23] L. E. Shelimova, O. G. Karpinskii, T. E. Svechnikova, E. S. Avilov, M. A. Kretova, and V. S. Zemskov, Inorg. Mater. 40, 1264 (2004).

[24] G. Kresse and J. Hafner, Phys. Rev. B 48, 13115 (1993).

[25] G. Kresse and J. Furthmüller, Comput. Mater. Sci. 6, 15 (1996).

[26] P. E. Blöchl, Phys. Rev. B 50, 17953 (1994).

[27] G. Kresse and D. Joubert, Phys. Rev. B 59, 1758 (1999).

[28] J. P. Perdew, K. Burke, and M. Ernzerhof, Phys. Rev. Lett. 77, 3865 (1996).

[29] A. A. Kordyuk, T. K. Kim, V. B. Zabolotnyy, D. V. Evtushinsky, M. Bauch, C. Hess, B. Buchner, H. Berger, and S. V. Borisenko, Phys. Rev. B 83, 081303(R) (2011).

[30] D. Pacilè, S. V. Eremeev, M. Caputo, M. Pisarra, O. De Luca, I. Grimaldi, J. Fujii, Z. S. Aliev, M. B. Babanly, I. Vobornik, R. G. Agostino, A. Goldoni, E. V. Chulkov, and M. Papagno, Phys. Status Solidi RRL 12, 1800341 (2018).

[31] I. P. Rusinov, I. A. Nechaev, and E. V. Chulkov, J. Exp. Theor. Phys. 116, 1006 (2013). 